\title{
An Empirical Study on the Use of Web 2.0 by Greek Adult Instructors in Educational Procedures
}

\author{
John Vrettaros, Alexis Tagoulis, Niki Giannopoulou, and Athanasios Drigas \\ NCSR DEMOKRITOS, \\ Institute of Informatics and Telecommunications \\ Net Media Lab \\ Ag. Paraskevi, 15310, Athens, Greece \\ \{dr,jvr\}@it.demokritos.gr, alextagoulis@hotmail.com, \\ ngianloy@gmail.com
}

\begin{abstract}
In this paper is presented an empirical study and its results. The empirical study was designed through a pilot training program which was conducted in order to learn if Greek educators can learn to use and even adopt the use of web 2.0 tools and services in the educational process and in which extend, where the type of learning is either distant learning, blended learning or the learning takes place in the traditional classroom.
\end{abstract}

Keywords: empirical study, Web 2.0, e-learning, social software, wikis, blogs, youtube, facebook.

\section{Introduction}

The web in the 90s helped to reduce the barriers regarding time, place and cultural boundaries, and contributed in the effective user communication and access to information. The philosophy behind web 2.0 , aims not only in the effective communication but also in the development of collaboration and networking of the users, which led for example to the creation of new terms like social networking. This evolution led to the transformation of learning, from the use e-learning platforms to the use of tools of the social web such as blogs, wikis, and forums, to a collaborative and cooperative learning framework. In this framework can be formed groups of learning in various levels of collaboration which can be small group, an organization, a society and finally the global community.

Since adult education is based in learning in groups, the new technologies of social networking can enhance learning effectiveness through the use of tools and services, with the appropriate learning scenarios, learning strategies, and adult education learning theories.

The technologies that support collaboration support the learning goal of changing stance related to learning based on learning groups. These technologies provide a reach, shared virtual learning space, where interactions are not taking place only between learns and technology but among learners, who share a mutual aim. These interactions can be facilitated and coordinated by an instructor. 
There have been many research and development efforts for the creation systems that support collaborative learning, but there is a research gap in how can we enhance learning effectiveness in adult education through the use of collaborative learning theories and techniques and collaborative tools and services provided by Web 2.0.

To be more specific, several web 2.0 tools have been widely used for collaborative learning purposes and the results have proved to be very fruitful. A wiki - based project was incorporated in a media technologies class where the learners developed the M/Cyclopedia (Media/Culture), a wiki - based encyclopedia, using MediaWiki of Wikipedia [1]. The educational use of wikis in distance learning classes has been analyzed in the context of a symbolic logic course [2] and wikis have also been used as an environment empowering cooperation among the distant learners [3]. Blogs have also been widely used in education. The creation of learning blogs has been attempted with the use of a group of 31 students that participated in an Information Systems class in Hong Kong University and this study finally proved, that blogs are a powerful tool when it comes to predicting final student performance [4]. Additionally, two groups (one consisted of professors and one consisted of students) were used in order for a research to be conducted aiming at determining the value of use of weblogs in education. The results made it more than clear that blogs were approved as a very useful tool in supporting physical presence in class [5]. Finally, Harvard University, Stanford University and Texas State University have set up virtual classes using Second Life via which learners participate in online courses. It is widely believed that Second Life can provide numerous possibilities when it comes to educational applications [6].

\section{Empirical Study}

For the wider and appropriate application of the web 2.0 tools and services in the learning process it is necessary the preparation of the adult trainers and also their opinion about these tools and services.

An approved way of investigating their stance and eliciting their opinions is testing these tools and services themselves, and take part in a learning process which comprises social networking tools and services. For the purpose described above an empirical study was designed which is described below

The aims is studying if the adult trainers have a positive stance against the web 2.0 tools and services, if they have used it, and if they can use the collaborative tools and services in an educational process designed by themselves.

Methodological Framework

Design of a learning community of practice

Formal characteristics

Year of realization of the training program: $2008-2009$

Place of realization: Greece

Training program title:

Technology used: LMS Moodle

Tools and services: social networking tools e.g. Blogs, wikis, social networking tools and services 
The degree of self-governance and the type of the training program, if it's a open one (learner participation in defining the learning process) or a closed one (there is little or no participation in defining the learning process)

The training program comprises features from open and closed type training programs, where the learners will be able to interfere and shape a new path for the learning process if they expect that it will be more effective in learning, not only in the training program core but also in the activities.

Training program content/curriculum

Aims of the model implementation:

- The learners will get to know others ways of learning

- The learners will explore e-commerce implementation

- The learners will be introduced to e-commerce and they will learn how can they develop a business activity

- The learners will explore the capabilities that e-commerce provides

- The learners will adopt a positive stance against the use of the new technologies in business activities.

Principles of the model implementation

The main principle that governs the community is the respect of the opinion of the peers. Every peer can freely think and express themselves. Through dialog the creative argument, the analysis and the synthesis of oppositions and positions is promoted. Through the interaction among peers and the collaboration of all stakeholders, proceed to aggregative actions.

\subsection{Process/ Methodological Approach}

\subsubsection{Actions/Methods of Instruction and Learning Process}

The actions considering the methods of instruction and learning will be based on the Project method. In the first phase the previous experiences and tacit knowledge of the learners will be explored, and the core of the training program will be configured.

In the second phase based on resources the educational material will be obtained. The learning groups will be defined and the activities or roles will be assigned to learning groups.

\subsubsection{Assessment (Assessment Criteria)}

The assessment criteria will be based on how useful the learners find the web 2.0 collaborative tools and services, and on how they can integrate e-commerce in their working life.

These questionnaires will be handed first, as a pilot to a smaller sample, for the respondents to co-design the questionnaire, in order to take their final form, which in this form the questionnaire will be handed the total sample.

Based on the statistical factor analysis that will follow, it is expected that important conclusions will be extracted, relevant to the factors significance considering the facilitation of the web 2.0 tools and services on learning, and in how these factors affect the learning effectiveness. Finally the correlation between these factors will be examined. 


\subsubsection{Process of Data Collection}

The trainees that participated in the survey got an invitation by email, which explicitly describes the form and the cause of the survey.

The questionnaire filling by the respondents took place electronically, and with the help of blogs there query elaboration took place in order to record every respondent question and remark.

Due to the fact that the training of the educators took place two months earlier than the survey, there are shots of the educational process accompanying the questionnaires, in order to help them recall easily the correspondent parts that are relevant to the survey. The respondents that took part in the survey are familiarized with the ICTs.

\subsubsection{Sample Description}

The sample consists of 30 learners who attended the realized training programs.

The demographic data of the correspondents are in our possession.

The sample consists of correspondents, aged between 30-55, who are adult trainers, certified that they have attended the National Train the Trainers Program. Their competences and skills in computer and internet use vary.

\subsection{Data Collection Tool}

The questionnaires consists of closed type questions, where in each question is asked the comment of the correspondent for each answer, in order to extract information that cannot be expressed in the answer itself.

The questionnaire is designed in decreasing Likert scale $(4=$ very much, $3=$ little, $2=$ average, $1=$ no)

The 4rth Likert degree was chosen in order to avoid having answers by undecided correspondents that usually express a medial position.

\subsubsection{Questionnaires}

Sex: Male, Female

Studies: Theoretical, Practical

Experience of work in Adult Education: Less than 5 years, 5 to 10 years, More than 10 years

\section{Before the training program}

Knowledge:

- Do you use e-learning platforms in teaching?

- Are you familiar with the tools below and do you know if they have implementations in teaching?

Competences:

- Are you familiarized with the use of tools and services of web 2.0? Stances:

- What's your opinion about the implementation of the web 2.0 tools and services in education? 
After the completion of the training program

- Was it difficult handling the web 2.0 tools and services, regarding the registration and the participation in the learning process?

- Did you need the help of an expert user to handle the web 2.0 tools and services?

- Based on the experienced acquired by your participation in the training program, what's your opinion about the educational value of the web 2.0 tools and services (blogs, wikis, youtube, facebook)?

- Do these tools satisfy the principles and the needs of adult education (blogs, wikis, youtube, facebook)?

- Do these tools lead to the elicitation of the tacit knowledge (blogs, wikis, youtube, facebook)?

- Do you think these tools can support learning by doing (blogs, wikis, youtube, facebook)?

- Do you think these tools can enhance collaborative learning (blogs, wikis, youtube, facebook)?

- Do you believe you can design and develop activities for adult education with the use of these tools (blogs, wikis, youtube, facebook)?

- (BLOG, WIKI, YOUTUBE)

- What's your opinion in the usefulness of facebook as a mean for the learners' first introduction?

- Do you think facebook can foster the creation of group atmosphere in a virtual class?

Knowledge:

- Do you think you acquired knowledge in using these tools and services (blogs, wikis, youtube, facebook)?

- Competences:

- Did you get familiarized with the use of these tools?

Stances:

- Would you use these tools in class (virtual or traditional)?

\section{Questionnaires Analysis}

In the first phase we analyzed the frequency of every question and some general remarks and in the second phase we will study the crossing of various questions (Crosstabs), and with the statistical check $\mathrm{X}^{2}$ possible causal relations.

For the first demographic data we didn't any further analysis, as the composition of the two training classes was such, that couldn't be found an equal allocation for the sexes, but the numbers statistically allow us to cross variables than weren't considered significant.

The second element, studies is artificial so there wasn't a question, but because the researcher knew the learners, divided them in these categories, and categorized as practical those who had previous experience in computers. 
Although the number of the correspondents is small and precarious to make crosstabs, it's very important because we study the effectiveness of tools and services of web 2.0 to non specialists in informatics educators.

In the research $75 \%$ of the educators has many years experience in adult education, as its shown by the answers in the $3^{\text {rd }}$ demographical question.

In the next question and after explaining to the correspondents that it is asked only the use of e-learning as an educator, a positive answer came from the practical educators, but they had all used e-learning as learners during their participation in the Training the Trainers Program.

The next question considers mainly their familiarization about the web 2.0 tools and services and their implementations in education. We observe that almost half of the educators know few about these tools, even the practical educators that have computer's experience knew few. Because the practical educators sample is small, we can have a secure estimation on that subject, and we must remark that there wasn't a negative answer in the familiarization with these tools.

From the four web 2.0 tools that they are asked for the most popular tool, the question concerns their opinion as simple users and not experts. In what concerns Facebook the answer a little means that they have browsed Facebook but they haven't created their own profile.

The last question is about their stances, which is quite determining for the survey, because its positive change during the training course and the survey indicates that the educators are ready to use the web 2.0 tools and services for educational purposes, and it also indicates the success of the pilot training program, not as a statistical but as an educational value.

Finally from a statistical point of view, the question is appropriate for $2 \chi$ checks and variable independence, because in every cross cell, we must have over five observations. On the specific question its distribution for all the categories is over five observations $(8.16,6)$, so we select this variable which is appropriate for crossing, for statistical and pedagogical reasons.

\section{After the completion of the pilot training program}

From this question we observe that most educators comprehend the function of the web 2.0 tools and services and its educational implementation without particular difficulties.

In this question we observe that a large percentage needed the help of an expert to use the web 2.0 tools and services, 63\%, which from a competences point of view leads as to the conclusion that the educators weren't ready to use the tools, from a competences point of view. Taking also under consideration the fact that $15 \%$ of the educators are specialized in informatics, we come to the conclusion that the educators' participation in other training programs in web 2.0 tools and services in education would help, for the successful implementation by the educators, in order to acquire the competences needed for the use of the tools and adoption of the use of the tools.

As we can see from the question concerning the educational value of the tools, only one respondent answered positively, where he has used the web 2.0 tools and services in class. 
Generally the respondents, 55\%-90\% have a more positive stance, after their participation in the training program, against the matters that affects the adult training.

One of the most important answers is the one that indicates that, through a short training program they gained the self efficacy, and the competences to develop their own learning activities through the use of the web2.0 tools and services (67\%).

Facebook became very popular among educators. Apparently, its use for the educators' acquaintance as trainees, which created a positive atmosphere in class, had an important role.

The educators' answers also show that their where greatly familiarized with the tools and services during the pilot training program, and recognized their educational value.

\section{Conclusions}

Through the empirical study presented we can understand the stances of Greek adult educators against the web 2.0 tools and services, but also the competences they can acquire through the use of the tools and services, but also the need for greater dissemination of the capabilities that the web tools and services in education. There is also the need for more training programs concerning web tools and services, and education in order, to be adopted by a large audience of educators.

An essential conclusion of this empirical study is that the trained educators, is that the use of the web 2.0 tools and services as its possible adoption in class by a large percentage of educators is generally easy and approachable even from educators that are not trained in ICTs.

\section{References}

1. Bruns, A., Humphreys, S.: Wikis in teaching and assessment: The M/Cyclopedia project. In: Proceedings of the 2005 International Symposium on Wikis, San Diego, CA, USA, October 16-18, pp. 25-32 (2005)

2. Byron, M.: Teaching with Tiki. Teaching Philosophy 28(2), 108-113 (2005)

3. Tsinakos, A.A.: Collaborative student modelling- A new perspective using wiki. WSEAS Transactions on Advances in Engineering Education 3(6), 475-481 (2006)

4. Du, H.S., Wagner, C.: Learning with weblogs: an empirical investigation. In: Proceedings of the 38th Hawaii International Conference on Systems Sciences (2005)

5. Barbosa, C.A.P., Serrano, C.A.: A blog as a construction tool for the cooperative learning and knowledge (2005)

6. Wongtangswad, J.: Uses of Second Life in Higher Education: Three Successful Cases. In: Richards, G. (ed.) Proceedings of World Conference on E-Learning in Corporate, Government, Healthcare, and Higher Education 2008, pp. 1389-1391. AACE, Chesapeake (2008) 